\title{
The effects of home-based stair and normal walking exercises on lower extremity functional ability, fall risk factors, and cardiovascular health risk factors in middle-aged older women
}

\author{
Suh-Jung Kang*, Chang-Hyun Ahn \\ Department of Sport \& Health Care, College of Art \& Culture, Sangmyung University, Seoul, Korea
}

The purpose of this study was to exam 12 weeks of stair and normal walking effects on lower extremity functional ability and cardiovascular health risk factors in middle-aged older women. Twenty-four subjects were assigned either to the stair walking group (SWG, $57.40 \pm 6.11$ years, $n=10$ ) or the normal walking group (NWG, $57.28 \pm 16.83$ years, $n=$ 14). They performed exercises 3 times per week, 60 min per day for 12 weeks. Lower extremity functional ability (lower extremity muscular strength, walking speed, active and static balance ability, and agility) and cardiovascular health risk factors were compared by time and groups using a two-way repeated analysis of variance and mean values were compared within group using paired $t$-test. As results, significant time differences were found in lower extremity muscular strength, walking speed and active balance; no time and group interactions were found. Also, significant time differences were found in systolic blood pressure, diastolic blood pressure, total cholesterol, triglycerides, fasting glucose. Significant time and group interactions were found in body fat. In addition, significant time differences within the groups were found in lower extremity muscular strength, walking speed, active balance in SWG and NWG; systolic blood pressure in SWG; systolic blood pressure, diastolic blood pressure, total cholesterol, triglycerides, and fasting glucose in NWG. These results indicated that home-based stair and normal walking exercises can be utilized to improve lower extremity functional ability and cardiovascular health risk factors in middle-aged and older women.

Keywords: Stain walking, Home-based exercise, Lower extremity functional ability, Fall risk factors

\section{INTRODUCTION}

Korea is now an aged society, and the country is expected to become a super-aged society as the percentage of the elderly population is predicted to reach $26 \%$ by 2026 . Such an increase in the old population has become a major social concern, as it can lead to health issues caused by chronic diseases and the consequent rise in medical expenses. It is natural that the human body becomes decrepit as a person ages; but as a result, we also see the deterioration of physical functions and an increase in the population with chronic diseases at the same time. Such chronic diseases are closely related to exercise habits and lifestyle.
Therefore, exercising is emphasized as a means of reducing health issues in the old population, and accordingly, various exercise programs are being used as part of an intervention strategy. Practicing exercise can provide the old population with such benefits such as delay in physiological changes caused by aging, optimization of body structure to be fitted with aging, pursuit of cognitive and psychological wellbeing, management of chronic diseases, lowered risk of disability, and prolonged lifespan. Specifically, the guidelines on physical activity for the old population recommend doing moderate exercises that do not cause any orthopedic stress at the frequency of 3-5 times per week, for at least 30$50 \mathrm{~min}$ per day, and for a total of $100 \mathrm{~min}$ per week. Such exercis-
${ }^{\star}$ Corresponding author: Suh-Jung Kang (D) https://orcid.org/0000-0002-8284-9299 Department of Sport \& Health Care, College of Art \& Culture, Sangmyung University, 7 Hongjimun 2ga-gil, Jongno-gu, Seoul 03015, Korea

E-mail: suhjkang@smu.ac.kr

Received: June 1, 2019 / Accepted: July 4, 2019
This is an Open Access article distributed under the terms of the Creative Commons Attribution Non-Commercial License (http://creativecommons.org/licenses/by-nc/4.0/) which permits unrestricted non-commercial use, distribution, and reproduction in any medium, provided the original work is properly cited. 
es include walking, underwater exercise, and stationary cycling (American College of Sports Medicine, 2014). In particular, walking is a physical activity that many people can do in general; it is highly accessible as people can easily enjoy walking and it also has a positive impact on improving physical strength (Kang, 2014; Murphy et al., 2007). Moreover, walking is easy and safe (Rafferty et al., 2002), and it shows a high sustained rate of participation in exercise (Hanson and Jones, 2015); therefore, it is one of the representative exercise programs that can be used for promoting people's health.

The previous studies reported that moderate walking exercise provides health benefits (Pate et al., 1995), it has a positive influence of cardiorespiratory fitness on patients with knee osteoarthritis (Larose et al., 2013). After doing a brisk walking exercise for 6 months, sedentary and physically deconditioned women over 60 years old showed improvements in walking endurance ability (Blain et al., 2017). Moreover, it was reported that regular walking activity with weight control reduced the incidence rate of metabolic syndrome in elderly Koreans (Kim and Kim, 2018). Walking, in those previous studies, was conducted on a flat-land outdoor environment, and it was observed that the effects of doing a walking-style exercise on a downhill road were also similar to those of walking on a flat-land. For instance, walking on a downhill road has the effect of enhancing the muscular strength of lower extremities (Gault et al., 2013; Rodio and Fattorini, 2014). In addition, it has been reported that a walking exercise like trekking, where uphill and downhill walking is mixed, has the effect of improving cardiovascular health and lower extremity muscular strength of obese old women (Kang, 2014).

Exercising provides a variety of health benefits, but people do not start or exercise for a long time due to environmental factors such as accessibility or convenience related to exercise facilities or spaces. Therefore, it is believed that home-based exercise can be an alternative solution to this problem as people can exercise on their own without the help of a professional instructor or use of any special sports equipment. However, it has been also reported that home-based exercise does not have any effect as people workout without an instructor or manager (Mediano et al., 2013). Moreover, there has been a report that no effect was observed after female participants, who were not obese, participated in moderate-intensity home-based exercise to enhance physical strength (Mediano et al., 2013). Recently, however, it was proved that home-based walking exercise is effective for those with peripheral vascular disease (Gardner et al., 2014); it was also known that home-based resistive exercise can enhance muscular strength and functional ability of old people (Thiebaud et al., 2014).

In this regard, this study aims to apply stair walking and normal walking exercises to middle-aged older women with a higher risk of cardiovascular disease, and then conduct comparative analyses regarding how such exercises can affect lower extremity functional ability, fall risk factors, and cardiovascular risk factors. The results of this study can be used as scientific evidence to verify the effect of stair walking that can be performed in people's residential areas and environments; results can also be used as basic data on the effectiveness of home-based walking exercises that people can do on their own without the help of professional sports trainer.

\section{MATERIALS AND METHODS}

\section{Participants}

The participants of this study were restricted to women without any orthopedic problem in the context of walking as an exercise, and who did not have experience in walking regularly for the previous 6 months. They were randomly divided into the stair walking group (SWG) and the normal walking group (NWG). At the beginning of the study, 11 people were recruited for SWG and 15 for NWG, but one person dropped out midway from each group, respectively, and therefore, the data of a total of 24 participants were used for the final analyses. Every participant signed a written consent form to participate in the study, and their physical characteristics are presented in Table 1.

\section{Exercise program}

\section{Stair walking program}

To explain the details and methods of stair walking, the researcher provided information on the exercise methods on the first day of the pretest. For stair walking, participants were guided to use the indoor stairs of the apartment building where they live or the office building where they work. During the first month, the participants repeated walking up and down the stairs for around fifth floors, and from the second month, when the participants became accustomed to stair walking, they repeated walking up and down

Table 1. Characteristics of subjects

\begin{tabular}{lcc}
\hline Characteristic & Age $(\mathrm{yr})$ & $\mathrm{BMl}\left(\mathrm{kg} / \mathrm{m}^{2}\right)$ \\
\hline SWG $(\mathrm{n}=10)$ & $57.4 \pm 6.11$ & $24.54 \pm 1.92$ \\
NWG $(\mathrm{n}=14)$ & $57.28 \pm 16.83$ & $24.76 \pm 4.18$ \\
Sig & 0.984 & 0.879 \\
\hline
\end{tabular}

Values are presented as mean \pm standard deviation. $\mathrm{BMI}$, body mass index; SWG, stair walking group; NWG, normal walking group. 
the stairs for around tenth or more floors. The total period of exercise was 12 weeks, and the exercise time per session was $60 \mathrm{~min}$ in total, during which 10 min were assigned for warm-up and cool-down, respectively; the walking exercise was carried out for $40 \mathrm{~min}$. The participants were directed to control the speed of exercise on their own at the intensity level of "slightly challenging," which corresponds to RPE-11-14 of the Borg scale. Moreover, to raise the exercise practicing rate of participants, exercise record notebooks were distributed on the day of the pretest, so that they could write down the date on which they do exercise and the start and end time of exercise. The research assistant collected the information once a week from every participant on the phone, and the exercise record notebooks were collected 12 weeks later.

\section{Normal walking program}

For normal walking, participants walked on walking trails in their neighborhood or at any other place where they could walk. As in the case of stair walking, the researcher provided information on the exercise methods on the first day of the pretest to offer guidance on the methods of normal walking, and exercise record notebooks were distributed. For normal walking, participants utilized pedestrian passages, parks, and walking trails in their neighborhood, and the total period, intensity, frequency, and exercise time per session were identical to those of stair walking. The manner in which participants were exercising was monitored on the phone as in the case of the SWG.

\section{Measuring method}

To analyze the effect of stair walking and normal walking on the lower extremity functional ability, fall risk factors, and cardiovascular risk factors, the same examiner measured the measurement variables under the same conditions before and after doing the walking exercise.

\section{Lower extremity functional ability and fall risk factors}

To assess lower muscular strength, participants carried out "sit and stand" and "squat against the wall" (Korea Sports Promotion Foundation, 2012). In doing "sitting on a chair and standing up," a folding stool was used, and the participants were directed to sit up straight on the center of the stool with their arms crossed in front of their chest. Then with a start signal, the number of times the participants repeated standing up and sitting on the chair for 30 sec was counted.

In doing "squat against the wall," the participants leaned the back against the wall with feet shoulder length apart; thereafter, they crossed their arms in front of their chest and bent knees at the height of sitting on a chair. The time for which the participants could maintain that posture was measured. In both tests, the participants did not speak while exercising to assess their accurate physical ability.

Participants walked at an ordinary speed for $10 \mathrm{~m}$ in a straight line in the gym, and they additionally walked for $5 \mathrm{~m}$ before and after the measuring section, respectively, to prevent walking speed from lowering at the beginning and the end part of walking. Walking speed $(\mathrm{sec} / \mathrm{m})$ was calculated by dividing the time taken to walk for a distance of $10 \mathrm{~m}$ by the distance walked (Shigematsu and Okura, 2006).

To assess balance, the dynamic balance and static balance tests were carried out. To test active balance, the participants carried out "running to and from a target $3 \mathrm{~m}$ away and returning to the chair" (Korea Sports Promotion Foundation, 2012). A cone-shaped target was put $3 \mathrm{~m}$ away from the stool; thereafter, the participants stood up as promptly as possible with the start signal, ran to and from the target, and sat down on the stool again. The time it took to do this and return to the stool once was measured, and the shorter record was adopted after measuring the time twice.

To assess static balance, the participants did the "standing on a single foot while closing eyes" task. The participants stood on a single foot while lifting up the other foot and spreading their arms wide to the side; the time they could maintain the balance on a single foot with eyes closed was measured.

To assess agility, the participants carried out "lying down and standing up." The participants were ordered to lay down straight on the mattress and then straightly stand up as promptly as possible. The number of times the participants could do this for $60 \mathrm{sec}$ was counted (Shigematsu and Okura, 2006).

\section{Cardiovascular risk factors}

Body fat percentage (\%) was measured by using a body composition analyzer (JEUS 9.0, Seoul, Korea); body mass index was calculated by using the measured height and weight of the participants. Waist circumference of the participants was measured as the narrowest part of the body, at the height of the waist between the ribs and the ilium, using a measuring tape. After the participants arrived and rested on a chair for at least 2 min to become relaxed, blood pressure was measured at the left upper arm by using a mercury sphygmomanometer. To take blood, participants were asked to keep the stomach empty for at least $12 \mathrm{hr}$ by fasting from 9:00 p.m. on the previous day of the test, as well as restricting physical activity at a moderate or intense level on the day of blood 
collection. Twenty milliliters of blood was collected from the brachial veins by a clinical laboratory expert using a disposable syringe. The collected blood was sent to and analyzed by a specialized blood analysis institution. From the blood lipids, total cholesterol (TC), high-density lipoprotein cholesterol, low-density lipoprotein cholesterol, triglycerides (TG), and fasting blood glucose (fasting glucose) were analyzed.

\section{Data analysis}

The data collected in this study were analyzed by using the IBM SPSS Statistics ver. 20.0 (IBM Co., Armonk, NY, USA) to calculate the mean and standard deviation of the entire data. A two-way repeated analysis of variance (ANOVA) was used to analyze the differences in the measured items (lower extremity functional ability, fall risk factors, and cardiovascular risk factors) after carrying out the two types of walking exercise (stair walking, normal walking) for 12 weeks, while a paired $t$-test was used to compare time differences within the groups. The statistical significance level was set at $\alpha=0.05$.

\section{RESULTS}

\section{Lower extremity functional ability and fall risk factors}

Table 2 displays the mean and standard deviation of lower extremity functional ability and fall risk factors before and after participating in walking exercises, and the comparative analysis results of the effect of exercise on these factors after exercising for 12 weeks. According to the ANOVA results, among the lower extremity muscular strength, significant differences were observed in sit and stand, squat against the wall, walking speed, and active balance factor after comparing the significant time difference before and after exercise for both groups together; no item showed significant time and group interaction effects. After carrying out paired $t$-test analyses of the time difference within the groups, significant differences were observed in the factors of lower strength, walking speed, active balance in SWG and NWG. Such results imply that it is possible to improve lower extremity muscular strength, walking speed, and balance by doing the two types of walking exercise for 12 weeks. However, the time and group interactions were not significant; this implies that there was no difference in the effect of exercise depending on the type of walking exercise, stair walking or normal walking.

\section{Cardiovascular risk factors}

Table 3 shows the mean and standard deviation of cardiovascular risk factors before and after participating in walking exercises and the comparative analysis results of the effect of exercise on these factors after exercising for 12 weeks. According to the ANOVA analysis results, significant differences were observed in the factors of systolic blood pressure (SBP), diastolic blood pressure (DBP), TC, TG, and fasting glucose after comparing the significant time difference before and after exercise for both groups together. Also, significant time and group interactions were observed in body fat. After carrying out paired $t$-test analyses of the

Table 2. Results of lower extremity functional fitness

\begin{tabular}{|c|c|c|c|c|c|c|c|}
\hline Variable & Group & Pre & Post & $\mathrm{T}$ (Sig) & Source & $F$ & Sig \\
\hline \multicolumn{8}{|c|}{ Lower extremity muscular strength } \\
\hline \multirow[t]{2}{*}{ Sit \& stand (reps) } & SWG & $11.10 \pm 1.59$ & $15.50 \pm 4.32$ & $-3.19\left(0.011^{*}\right)$ & $\mathrm{T}$ & 15.941 & $0.001^{*}$ \\
\hline & NWG & $14.30 \pm 3.96$ & $17.07 \pm 3.45$ & $-2.374\left(0.035^{*}\right)$ & $T \times G$ & 0.825 & 0.374 \\
\hline \multirow[t]{2}{*}{ Squat wall (reps) } & SWG & $39.28 \pm 20.58$ & $72.79 \pm 46.28$ & $-2.249\left(0.051^{*}\right)$ & $\mathrm{T}$ & 16.337 & $0.001^{*}$ \\
\hline & NWG & $39.34 \pm 30.73$ & $105.0 \pm 68.54$ & $-3.615\left(0.004^{*}\right)$ & $T \times G$ & 1.716 & 0.204 \\
\hline \multirow[t]{2}{*}{ Walking speed (sec/m) } & SWG & $7.56 \pm 0.31$ & $5.65 \pm 0.95$ & $5.604\left(0.000^{*}\right)$ & $\mathrm{T}$ & 58.990 & $0.000^{*}$ \\
\hline & NWG & $7.35 \pm 0.71$ & $6.20 \pm 0.61$ & $4.960\left(0.000^{*}\right)$ & $T \times G$ & 3.634 & 0.070 \\
\hline \multicolumn{8}{|l|}{ Balance (sec) } \\
\hline \multirow[t]{2}{*}{ Active balance } & SWG & $7.57 \pm 1.05$ & $6.06 \pm 0.97$ & $6.504\left(0.000^{*}\right)$ & $\mathrm{T}$ & 12.869 & $0.002^{*}$ \\
\hline & NWG & $6.88 \pm 1.45$ & $5.06 \pm 2.48$ & $2.298\left(0.040^{*}\right)$ & $T \times G$ & 0.106 & 0.748 \\
\hline \multirow[t]{2}{*}{ Static balance } & SWG & $17.14 \pm 15.98$ & $19.76 \pm 15.04$ & $-0.430(0.677)$ & $\mathrm{T}$ & 0.620 & 0.440 \\
\hline & NWG & $16.46 \pm 16.85$ & $18.37 \pm 19.0$ & $-0.979(0.347)$ & $T \times G$ & 0.015 & 0.903 \\
\hline \multirow[t]{2}{*}{ Agility (reps) } & SWG & $16.65 \pm 4.14$ & $17.40 \pm 4.42$ & $-1.256(0.241)$ & $\mathrm{T}$ & 1.324 & 0.263 \\
\hline & NWG & $14.67 \pm 7.01$ & $15.56 \pm 5.20$ & $-0.145(0.887)$ & $T \times G$ & 0.009 & 0.924 \\
\hline
\end{tabular}

Values are presented as mean \pm standard deviation.

SWG, stair walking group; NWG, normal walking group; Sit \& stand, sitting on a chair and standing up; Squat wall, squat against the wall; T, time; G, group.

${ }^{*} P<0.05$, statistically significant differences. 
Table 3. Results of cardiovascular health risk factors

\begin{tabular}{|c|c|c|c|c|c|c|c|}
\hline Variable & Group & Pre & Post & $\mathrm{T}(\mathrm{Sig})$ & Source & $F$ & Sig \\
\hline \multirow[t]{2}{*}{ Body fat (\%) } & SWG & $33.22 \pm 2.19$ & $33.83 \pm 1.77$ & $-2.781\left(0.021^{*}\right)$ & $\mathrm{T}$ & 0.019 & 0.892 \\
\hline & NWG & $33.22 \pm 6.35$ & $32.50 \pm 5.58$ & $1.103(0.292)$ & $T \times G$ & 2.982 & $0.009^{*}$ \\
\hline \multirow[t]{2}{*}{$\mathrm{BMI}\left(\mathrm{kg} / \mathrm{m}^{2}\right)$} & SWG & $24.54 \pm 1.92$ & $24.86 \pm 1.68$ & $-2.027(0.073)$ & $\mathrm{T}$ & 0.420 & 0.524 \\
\hline & NWG & $24.31 \pm 3.99$ & $24.23 \pm 3.90$ & 0.274 (0.789) & $\mathrm{T} \times \mathrm{G}$ & 1.190 & 0.288 \\
\hline \multirow[t]{2}{*}{ WC (cm) } & SWG & $81.92 \pm 9.02$ & $81.92 \pm 7.59$ & $-0.270(0.793)$ & $\mathrm{T}$ & 0.067 & 0.798 \\
\hline & NWG & $83.93 \pm 5.81$ & $84.30 \pm 4.27$ & $0.000(1.000)$ & $T \times G$ & 0.067 & 0.798 \\
\hline \multirow[t]{2}{*}{$\mathrm{SBP}(\mathrm{mmHg})$} & SWG & $126.0 \pm 8.09$ & $117.5 \pm 12.74$ & $2.375\left(0.042^{*}\right)$ & $\mathrm{T}$ & 9.319 & $0.006^{*}$ \\
\hline & NWG & $142.30 \pm 17.27$ & $128.46 \pm 24.78$ & $2.396\left(0.034^{*}\right)$ & $T \times G$ & 0.533 & 0.473 \\
\hline \multirow[t]{2}{*}{$\mathrm{DBP}(\mathrm{mmHg})$} & SWG & $78.0 \pm 7.52$ & $72.50 \pm 8.24$ & $2.181(0.057)$ & $\mathrm{T}$ & 11.616 & $0.003^{*}$ \\
\hline & NWG & $85.38 \pm 10.50$ & $80.76 \pm 8.37$ & $2.650\left(0.021^{*}\right)$ & $T \times G$ & 0.089 & 0.769 \\
\hline \multirow[t]{2}{*}{$\mathrm{TC}(\mathrm{mg} / \mathrm{dL})$} & SWG & $209.60 \pm 34.93$ & $195.50 \pm 34.68$ & $2.025(0.074)$ & $\mathrm{T}$ & 11.036 & $0.003^{*}$ \\
\hline & NWG & $225.38 \pm 38.30$ & $205.50 \pm 25.96$ & $2.721\left(0.020^{*}\right)$ & $T \times G$ & 0.330 & 0.572 \\
\hline \multirow[t]{2}{*}{$\mathrm{HDL}-\mathrm{C}(\mathrm{mg} / \mathrm{dL})$} & SWG & $58.60 \pm 8.51$ & $62.40 \pm 11.71$ & $-2.246(0.051)$ & $\mathrm{T}$ & 3.560 & 0.074 \\
\hline & NWG & $64.15 \pm 15.58$ & $70.41 \pm 14.61$ & $-1.226(0.246)$ & $T \times G$ & 0.044 & 0.836 \\
\hline \multirow[t]{2}{*}{ LDL-C (mg/dL) } & SWG & $125.90 \pm 32.92$ & $121.90 \pm 32.37$ & $0.548(0.597)$ & $\mathrm{T}$ & 2.278 & 0.147 \\
\hline & NWG & $133.46 \pm 37.78$ & $122.41 \pm 26.23$ & $1.671(0.123)$ & $T \times G$ & 0.445 & 0.513 \\
\hline \multirow[t]{2}{*}{$\mathrm{TG}$ (mg/dL) } & SWG & $149.5 \pm 52.23$ & $125.0 \pm 31.55$ & $2.069(0.068)$ & $\mathrm{T}$ & 7.754 & $0.011^{*}$ \\
\hline & NWG & $148.0 \pm 57.96$ & $97.16 \pm 44.23$ & $2.197\left(0.050^{*}\right)$ & $T \times G$ & 0.671 & 0.422 \\
\hline \multirow[t]{2}{*}{ Fasting glucose (mg/dL) } & SWG & $93.3 \pm 13.52$ & $88.90 \pm 12.36$ & $1.848(0.098)$ & $\mathrm{T}$ & 9.440 & $0.006^{*}$ \\
\hline & NWG & $99.07 \pm 5.51$ & $94.0 \pm 8.40$ & $2.533\left(0.028^{*}\right)$ & $T \times G$ & 0.149 & 0.703 \\
\hline
\end{tabular}

Values are presented as mean \pm standard deviation.

BMI, body mass index; WC, waist circumference; SBP, systolic blood pressure; DBP, diastolic blood pressure; TC, total cholesterol; HDL-C, high-density lipoprotein cholesterol; LDL-C, low-density lipoprotein cholesterol; TG, triglyceride; SWG, stair walking group; NWG, normal walking group; T, time; G, group.

${ }^{*} P<0.05$, statistically significant differences.

time difference within the groups, significant differences were observed in the factors of body fat, SBP in SWG; SBP, DBP, TC, TG, fasting glucose in NWG. Such results suggest that it is possible to improve body fat, SBP, DBP, TC, TG, and fasting glucose by performing the two types of walking exercise for 12 weeks. More specifically, it also means that stair walking can improve body fat percentage and SBP, while normal walking can improve the factors of SBP, DBP, TC, TG, and fasting glucose. However, the time and group interactions were found only in body fat; this implies that 12 weeks of walking exercise can result in positive effects but the effect of exercise depending on the type of walking exercise, stair walking or normal walking is minimal.

\section{DISCUSSION}

The purpose of this study was to analyze the impact of two types of a home-based walking exercise program on the lower extremity functional ability, fall risk factors, and cardiovascular risk factors of middle-aged older women; the discussion on the major results are as follows.

First, among the lower extremity functional ability, significant time differences were observed in lower extremity muscular strength, walking speed, and active balance for both groups. However, the effect of the time and group interactions was not significant. In other words, regularly doing the two types of walking exercise for 12 weeks could be considered as an effective exercise program to improve lower extremity functional ability, walking speed, and balance, but it means that there was no difference in the effect depending on the type of walking exercise. Such results show a similar trend to the results of a review study (Thiebaud et al., 2014) that analyzed the effect of home-based resistive exercises on the elderly. This study has its limitations, as it did not measure muscle mass to analyze the effect on lower extremity functional ability and fall risk factors. Based on the results of a previous study (Newman et al., 2006) that the level of muscular strength can be enhanced even without any change in muscle mass, the enhanced lower extremity muscular strength observed in this study can be regarded as a significant outcome. Moreover, considering the fact that the deterioration of muscular strength (Newman et al., 2006) and the deterioration of lower extremity muscular endurance (Manini et al., 2007) are the predictors of mortality risk, it can be said that stair walking and normal walking have significance in relation to the 
disability adjusted life expectancy.

As for walking speed, a significant time difference was observed in both groups, and there was no difference in the effect depending on the type of exercise program. Such a result provides very important implications. It was recently reported that slower walking speed is an important sign that one's health condition deteriorates along with aging and disease (Studenski, 2009), and slower walking speed is significantly related to cognitive function and the risk of dementia (Quan et al., 2017). Moreover, walking speed is an independent factor that predicts the starting point of deterioration in the ability of the instrumental activity of daily living (Makizako et al., 2015). It is also a very important physical ability, which is related to the disability and fall frequency of elderly people (Nourhashémi et al., 2001). In general, slower walking speed is a commonly observed symptom that can be accompanied by decreasing muscular strength of elderly people, and as a result, it can be said to be a major factor that undermines the functional independence of an individual person. Mobility, related to walking, is another major factor that is related with quality of life and functional independence, and it has been reported that $35 \%$ of disabled people in the United States are affected by mobility-related concerns (Courtney-Long et al., 2015). Therefore, this study presented the likelihood of lowering health risks for middle-aged and elderly women by doing home-based exercises using stairs, without visiting any special exercise facilities or without the help of professional exercise instructor.

Second, among the cardiovascular risk factors, body fat percentage, SBP, DBP, TC, TG, and fasting glucose showed a significant time difference within the groups, and significant interaction was observed in body fat. Such a result also shows that the two types of walking exercise are effective in addressing cardiovascular health risks. In this study, it was observed that stair walking was more effective in controlling obesity than normal walking, because in the case of body fat percentage, there was a significant time difference only in the SWG. This study did not measure the energy consumption during the two types of walking exercise, and the participants controlled the intensity of exercise on their own by rating perceived exertion on the Borg scale. It seems that body fat percentage showed a significant decrease only in the SWG because going up and down the stairs probably caused the participants to consume more energy.

Obesity negatively affects the health-related quality of life (HRQoL; Kolotkin et al., 2001), and the reduction of body composition factors has importance as it can improve HRQoL, physical function, general health, vitality, and mental health (Fontaine et al., 1999). More importantly, the decrease in body composition lowers the risk of obesity-related complications such as high blood pressure, cardiovascular diseases, vessel diseases, and type II diabetes, so it can be said that the effect of stair walking exercise has a very important meaning.

Blood pressure and blood lipids are among cardiovascular risk factors. In this study, SBP showed a significant difference in both groups, while DBP showed a significant difference in the NWG. High blood pressure is a major risk factor of cardiovascular disease (Egan et al., 2010), and by lowering SBP by $2 \mathrm{mmHg}$, the death rate from stroke and ischemic heart disease can be lowered by $10 \%$ and $7 \%$, respectively (Lewington et al., 2002). Therefore, this study presented very important implications in the sense that even microscopic changes can be evaluated as valuable clinical changes. Along with high blood pressure, type II diabetes is a major disease that can develop into cardiovascular disease (Joseph and Golden, 2014). Patients with type II diabetes have a shorter life expectancy than healthy people, and in this case, the risk of cardiovascular disease and death rate from any cause of death are 2-4 times higher (Rao Kondapally Seshasai et al., 2011; Sattar, 2013). With regard to this, according to the results of a recently completed study, the results of a 10-year follow-up study of 1,198 patients with type II diabetes showed that the risk of death rate from any cause of death was most affected by fasting glucose and high blood pressure (Afsharian et al., 2016). The risk of cardiovascular disease was most affected by levels of fasting glucose. The results of a meta-analysis conducted by compiling and analyzing the previous studies on the effect of walking exercise to improve the aforementioned factors showed that walking exercise could positively affect aerobic fitness, including blood pressure and blood lipids components, and blood pressure and body fat percentage (Murphy et al., 2007; Murtagh et al., 2015). According to the results of the present study, the effect on body fat percentage or SBP was observed in the SWG, while an improvement in blood lipids and fasting glucose was mainly observed in the NWG. Therefore, the results of this study can be used as supporting data for the intervention strategy to instruct people to use the appropriate type of walking exercise in consideration of health issues of an individual person.

Third, people do not feel any financial burden with a home-based exercise program, and the program has the merit of convenience because people can work out without the help of professional exercise instructors (McDermott and Polonsky, 2016). Moreover, there is no issue of availability of exercise coaching depending on whether it is covered by the national insurance or not, and there is 
no case where people cannot work out as they feel emotional pressure to go to a sports facility (Harwood et al., 2016). This study provided general education before starting the home-based exercise program so that participants could exercise on their own and also conducted monitoring via the phone to help people keep exercising. As a result, the two types of walking exercise showed positive results. This shows that a home-based walking exercise can be used as an effective exercise method of health management for those who cannot work out with a professional exercise instructor. In conclusion, this study demonstrated that a 12-week homebased regular stair climbing and flat ground normal walking exercise without an instructor provided improvements in lower extremities functional fitness, fall risk factors, and cardiorespiratory risk factors. However, these effects were not different by walking modes. These findings suggest that middle-aged older women may consider home-based stair climbing and walking to prevent age-related chronic diseases and improve HRQoL.

This study has the following limitations: First, as it was hard to recruit a sufficient number of participants, statistical significance in some factors of measurement items was not obtained. Therefore, ex-post facto research needs to be conducted based on the results of this study with more participants for further in-depth study. Second, the average age of participants in both groups was in the late 50s. Therefore, the possibility that the measurement results were overestimated could not be completely ruled out because this study used measuring methods developed for the elderly to assess lower extremity functional ability and fall risk factors. Third, a control group to analyze the effect of walking exercise was not constituted; therefore, this study was restricted to the analysis of the difference between stair walking and normal walking.

If future research uses tools such as a pedometer or a physical activity meter to present specific guidelines on stair walking exercise for the elderly based on the results of this study, more specific guidelines can be presented regarding the recommended intensity and level of stair walking exercise. Moreover, it would be very meaningful to study the effect of various types of home-based walking exercises on cognitive functioning of the elderly, based on the results of previous studies that older people in Korea show a higher participation rate in walking exercise and that such exercise is effective in improving cognitive function.

\section{CONFLICT OF INTEREST}

No potential conflict of interest relevant to this article was reported.

\section{REFERENCES}

Afsharian S, Akbarpour S, Abdi H, Sheikholeslami F, Moeini AS, Khalili D, Momenan AA, Azizi F, Hadaegh F. Risk factors for cardiovascular disease and mortality events in adults with type 2 diabetes - a 10-year follow-up: Tehran Lipid and Glucose Study. Diabetes Metab Res Rev 2016;32:596-606.

American College of Sports Medicine. ACSM's resource manual for guidelines for exercise testing and prescription. 7th ed. Philadelphia (PA): Lippincott Williams \& Wilkins; 2014.

Blain H, Jaussent A, Picot MC, Maimoun L, Coste O, Masud T, Bousquet J, Bernard PL. Effect of a 6-month brisk walking program on walking endurance in sedentary and physically deconditioned women aged 60 or older: a randomized trial. J Nutr Health Aging 2017;21:11831189

Courtney-Long EA, Carroll DD, Zhang QC, Stevens AC, Griffin-Blake S, Armour BS, Campbell VA. Prevalence of disability and disability type among adults-United States, 2013. MMWR Morb Mortal Wkly Rep 2015;64:777-783.

Egan BM, Zhao Y, Axon RN. US trends in prevalence, awareness, treatment, and control of hypertension, 1988-2008. JAMA 2010;303:20432050.

Fontaine KR, Barofsky I, Andersen RE, Bartlett SJ, Wiersema L, Cheskin LJ, Franckowiak SC. Impact of weight loss on health-related quality of life. Qual Life Res 1999;8:275-277.

Gardner AW, Parker DE, Montgomery PS, Blevins SM. Step-monitored home exercise improves ambulation, vascular function, and inflammation in symptomatic patients with peripheral artery disease: a randomized controlled trial. J Am Heart Assoc 2014;3:e001107.

Gault ML, Clements RE, Willems MET. Cardiovascular responses during downhill treadmill walking at self-selected intensity in older adults. J Aging Phys Act 2013;21:335-347.

Hanson S, Jones A. Is there evidence that walking groups have health benefits? A systematic review and meta-analysis. Br J Sports Med 2015;49:710-715.

Harwood AE, Smith GE, Cayton T, Broadbent E, Chetter IC. A Systematic review of the uptake and adherence rates to supervised exercise programs in patients with intermittent claudication. Ann Vasc Surg 2016;34:280-289.

Joseph JJ, Golden SH. Type 2 diabetes and cardiovascular disease: what next? Curr Opin Endocrinol Diabetes Obes 2014;21:109-120.

Kang SJ. Trekking exercise promotes cardiovascular health and fitness benefits in older obese women. J Exerc Rehabil 2014;10:225-229.

Kim S, Kim DI. Association of regular walking and body mass index on metabolic syndrome among an elderly Korean population. Exp Geron- 
tol 2018;106:178-182.

Kolotkin RL, Meter K, Williams GR. Quality of life and obesity. Obes Rev 2001;2:219-229.

Korea Sports Promotion Foundation. Fitness promotion guideline for elderly. Ministry of Culture, Sports, and Tourism; 2012.

Larose J, King J, Brosseau L, Wells GA, Reid R, Maetzel A, Tugwell P, Huijbregts M, McCullough C, Loew L, Kenny GP. The effect of walking on cardiorespiratory fitness in adults with knee osteoarthritis. Appl Physiol Nutr Metab 2013;38:886-891.

Lewington S, Clarke R, Qizilbash N, Peto R, Collins R; Prospective Studies Collaboration. Age-specific relevance of usual blood pressure to vascular mortality: a meta-analysis of individual data for one million adults in 61 prospective studies. Lancet 2002;360:1903-1913.

Makizako H, Shimada H, Doi T, Tsutsumimoto K, Lee S, Hotta R, Nakakubo S, Harada K, Lee S, Bae S, Harada K, Suzuki T. Cognitive functioning and walking speed in older adults as predictors of limitations in self-reported instrumental activity of daily living: prospective findings from the Obu Study of Health Promotion for the Elderly. Int J Environ Res Public Health 2015;12:3002-3013.

Manini TM, Visser M, Won-Park S, Patel KV, Strotmeyer ES, Chen H, Goodpaster B, De Rekeneire N, Newman AB, Simonsick EM, Kritchevsky SB, Ryder K, Schwartz AV, Harris TB. Knee extension strength cutpoints for maintaining mobility. J Am Geriatr Soc 2007;55:451-457.

McDermott MM, Polonsky TS. Home-based exercise: a therapeutic option for peripheral artery disease. Circulation 2016;134:1127-1129.

Mediano MF, Neves FA, Cunha AC, Souza EP, Moura AS, Sichieri R. Changes in body weight, C-reactive protein, and total adiponectin in non-obese women after 12 months of a small-volume, home-based exercise program. Clinics (Sao Paulo) 2013;68:1121-1127.

Murphy MH, Nevill AM, Murtagh EM, Holder RL. The effect of walking on fitness, fatness and resting blood pressure: a meta-analysis of randomised, controlled trials. Prev Med 2007;44:377-385.

Murtagh EM, Nichols L, Mohammed MA, Holder R, Nevill AM, Murphy $\mathrm{MH}$. The effect of walking on risk factors for cardiovascular disease: an updated systematic review and meta-analysis of randomised control trials. Prev Med 2015;72:34-43.
Newman AB, Kupelian V, Visser M, Simonsick EM, Goodpaster BH, Kritchevsky SB, Tylavsky FA, Rubin SM, Harris TB. Strength, but not muscle mass, is associated with mortality in the health, aging and body composition study cohort. J Gerontol A Biol Sci Med Sci 2006;61:72-77.

Nourhashémi F, Andrieu S, Gillette-Guyonnet S, Vellas B, Albarède JL, Grandjean $\mathrm{H}$. Instrumental activities of daily living as a potential marker of frailty: a study of 7364 community-dwelling elderly women (the EPIDOS study). J Gerontol A Biol Sci Med Sci 2001;56:M448-453.

Pate RR, Pratt M, Blair SN, Haskell WL, Macera CA, Bouchard C, Buchner D, Ettinger W, Heath GW, King AC. Physical activity and public health. A recommendation from the Centers for Disease Control and Prevention and the American College of Sports Medicine. JAMA 1995;273:402-407.

Quan M, Xun P, Chen C, Wen J, Wang Y, Wang R, Chen P, He K. Walking pace and the risk of cognitive decline and dementia in elderly populations: a meta-analysis of prospective cohort studies. J Gerontol A Biol Sci Med Sci 2017;72:266-270.

Rafferty AP, Reeves MJ, McGee HB, Pivarnik JM. Physical activity patterns among walkers and compliance with public health recommendations. Med Sci Sports Exerc 2002;34:1255-1261.

Rao Kondapally Seshasai S, Kaptoge S, Thompson A, Di Angelantonio E, Gao P, Sarwar N, Whincup PH, Mukamal KJ, Gillum RF, Holme I, Njølstad I, Fletcher A, Nilsson P, Lewington S, Collins R, Gudnason V, Thompson SG, Sattar N, Selvin E, Hu FB, Danesh J; Emerging Risk Factors Collaboration. Diabetes mellitus, fasting glucose, and risk of cause-specific death. N Engl J Med 2011;364:829-841.

Rodio A, Fattorini L. Downhill walking to improve lower limb strength in healthy young adults. Eur J Sport Sci 2014;14:806-812.

Sattar N. Revisiting the links between glycaemia, diabetes and cardiovascular disease. Diabetologia 2013;56:686-695.

Shigematsu R, Okura T. A novel exercise for improving lower-extremity functional fitness in the elderly. Aging Clin Exp Res 2006;18:242-248.

Studenski S. Bradypedia: is gait speed ready for clinical use? J Nutr Health Aging 2009;13:878-880.

Thiebaud RS, Funk MD, Abe T. Home-based resistance training for older adults: a systematic review. Geriatr Gerontol Int 2014;14:750-757. 